\title{
On Planning for Whole Arm Manipulation with Switching Contact Modes
}

\author{
Masahito Yashima \\ National Defense Academy, Yokosuka 239-8686 JAPAN \\ yashima@nda.ac.jp
}

\begin{abstract}
This paper presents mathematical conditions and algorithm for a whole arm manipulation planning with switching contact modes. The motion planning for such a system should consider changing the dynamics and kinematics according to contact modes. We show the conditions about kinematic and dynamics aspects to be satisfied for the motion planning. The conditions give the restriction of feasible contact modes and the number of contact points. The planning problem is simplified using a randomized technique. We illustrate the approach by simulating the whole arm manipulation.
\end{abstract}

\section{Introduction}

In contrast to fingertip grasps, whole arm grasps are formed by enveloping the arms around objects. There are many tasks such as lifting up heavy loads or constraining large objects that necessitate the whole arm grasps. While there are many works which consider whole arm grasps, most of them are contact force analyses of power grasps and their robustness in order to firmly grasp objects which do not move relative to the arms[10],[17]. On the other hand, our interests are to plan manipulating objects using the inner links of the arms in order to move the objects to a goal configuration. Such manipulation is called whole arm manipulation. There are some works concerning whole arm manipulation by Yashima[11],[15],Trinkle[12], Kaneko[4].

Motion planning for the manipulation by a multifingered robot hand is a complicated problem since a number of the arms are involved but rather because of the nature of the contact such as rolling and sliding. Most of works deal with instantaneous or local motion planning, and assume that there are not any change of contact modes between the arms and objects during tasks. In contrast to the conventional manipulation, we consider the manipulation with changing contact modes so that we can achieve a dextrous manipulation. In this case, we should take into consideration for the manipulation systems with changing dynamics according to the change of contact modes and verify the kinematical and dynamical characteristics of such manipulation systems. Li[7] studied instantaneous kinematic manipulation plan- ning by considering different contact modes. Trinkle[13] showed a global motion planning of dextrous manipulation with frictionless contacts. Cherif[1] presented an algorithm of global motion planning for quasi-static fingertip manipulation. In these studies, the manipulation is restricted to a quasi-static motion.

For a system with changing dynamics behavior, the state space of manipulation systems consists of multiple partitions which correspond to a contact mode. Each partition is governed by a different set of differential equations. In order to complete a successful manipulation, it is necessary to plan transitions of the contact modes. The systems which can be modeled by discrete event such as a contact mode and continuous dynamics is called hybrid systems[6]. Many studies about the hybrid systems are the stability and modeling from the view of control theory. Zefran[16] applied the idea of hybrid systems to the study of grasp gaits. To my knowledge, there is no study about manipulation with switching contacts.

In this paper, we first develop a mathematical conditions to be satisfied for motion planning, based on the kimenatics and dynamics of manipulation systems. These conditions give the restriction for feasible contact modes and the number of contact points. We then describe the basic ideas of manipulation planning, which is defined as 'given initial and goal object states, it is to find a joint torque trajectory, using rolling and sliding contacts'. The proposed algorithm is simplified using a randomized technique. Finally we apply the ideas to the simulation of a whole arm manipulation system.

\section{Problem formulation}

We consider a planar whole arm manipulation system which is subject to Coulomb's friction as shown in Figure 1. We assume that each link of each arm (hand) has one contact point with an object. Each joint has one degree of freedom. Suppose there are $n_{C}$ contacts, consisting of $n_{R}$ rolling contacts and $n_{S}$ sliding contacts. Let the subscripts $N$ and $T$ denote the inward normal and tangential directions at the contact point on object's surface, and $S$ and $R$ denote sliding and rolling contacts, respectively. We denote $i$ th contact point by subscript $i$. 


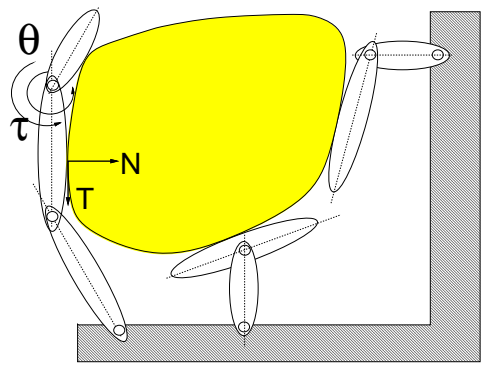

Figure 1: General whole arm manipulation.

The dynamic equations of rigid object can be written as

$$
\boldsymbol{M}_{o} \ddot{\boldsymbol{q}}=\boldsymbol{G}_{A \mu} \boldsymbol{f}_{A}+\boldsymbol{g}_{o}
$$

where

$\boldsymbol{G}_{A \mu}=\left[\boldsymbol{G}_{N S}+\boldsymbol{G}_{T S} \boldsymbol{U}_{S}, \boldsymbol{G}_{N R}, \boldsymbol{G}_{T R}\right]$,

$\boldsymbol{f}_{A}^{T}=\left[\boldsymbol{f}_{N S}^{T}, \boldsymbol{f}_{N R}^{T}, \boldsymbol{f}_{T R}^{T}\right]$,

$\boldsymbol{U}_{S}=\operatorname{diag}\left[\cdots,-\operatorname{sgn}\left(v_{i T S}\right) \mu_{i S}, \cdots\right], \quad \ddot{\boldsymbol{q}}$ is the 3-vector of linear and angular acceleration of the center of mass, $\boldsymbol{M}_{o}$ is the $3 \times 3$-mass matrix of the object, $\boldsymbol{g}_{o}$ is external wrench applied to the object, $v_{i T S}$ is sliding velocity, and $\mu_{i S}$ is the coefficient of kinetic friction. $\boldsymbol{f}_{N S}$ is the $n_{S}$-vector of normal forces at sliding contacts, while $\boldsymbol{f}_{N R}$ and $\boldsymbol{f}_{T R}$ are the $n_{R}$-vectors of normal and tangential forces at rolling contacts, respectively. $\boldsymbol{G}_{N S}$ and $\boldsymbol{G}_{T S}$ are $3 \times n_{S}$ wrench matrices in the normal and tangential direction for sliding contacts. $\boldsymbol{G}_{N R}$ and $\boldsymbol{G}_{T R}$ are $3 \times n_{R}$ wrench matrices in the normal and tangential direction for rolling contacts.

The motion equation of the arms can be written as

$$
\boldsymbol{M}_{a} \ddot{\boldsymbol{\theta}}=\boldsymbol{\tau}-\boldsymbol{J}_{A \mu}^{T} \boldsymbol{f}_{A}-\boldsymbol{g}_{a}
$$

where

$\boldsymbol{J}_{A \mu}^{T}=\left[\boldsymbol{J}_{N S}^{T}+\boldsymbol{J}_{T S}^{T} \boldsymbol{U}_{S}, \boldsymbol{J}_{N R}^{T}, \boldsymbol{J}_{T R}^{T}\right]$, and $\boldsymbol{J}_{N S}, \boldsymbol{J}_{T S}$, $\boldsymbol{J}_{N R}$, and $\boldsymbol{J}_{T R}$ are hand Jacobians defined like the wrench matrices. $\ddot{\boldsymbol{\theta}}$ is the $n_{\theta}$-vector of joint accelerations, and $\boldsymbol{M}_{a}$ is the $n_{\theta} \times n_{\theta}$-inertia matrix of the arm. $\boldsymbol{\tau}$ is the $n_{\theta}$-vector of joint control torque. $\boldsymbol{g}_{a}$ is the vector of torque caused by external wrenches and velocity product wrenches.

The Coulomb's friction law can be written as

$$
\boldsymbol{B}_{\mu} \boldsymbol{f}_{A} \geq \mathbf{0}
$$

where $\boldsymbol{U}_{R}=\operatorname{diag}\left[\cdots,-\mu_{i R}, \cdots\right], \boldsymbol{E}_{S}$ and $\boldsymbol{E}_{R}$ are $n_{S} \times n_{S}$ and $n_{R} \times n_{R}$ unit matrices, and $\mu_{i R}$ is the coefficient of static friction, $\boldsymbol{B}_{\mu}=\left[\begin{array}{ccc}\boldsymbol{E}_{S} & \multicolumn{2}{c}{\mathbf{0}} \\ \mathbf{0} & \boldsymbol{U}_{R} & \boldsymbol{E}_{R} \\ & \boldsymbol{U}_{R} & -\boldsymbol{E}_{R}\end{array}\right]$.

The physically admissible contact motion for rolling and sliding contacts can be formulated as

$$
\boldsymbol{G}_{A}^{T} \dot{\boldsymbol{q}}=\boldsymbol{J}_{A} \dot{\boldsymbol{\theta}}
$$

where

$\boldsymbol{G}_{A}=\left[\boldsymbol{G}_{N S}, \boldsymbol{G}_{N R}, \boldsymbol{G}_{T R}\right], \boldsymbol{J}_{A}^{T}=\left[\boldsymbol{J}_{N S}^{T}, \boldsymbol{J}_{N R}^{T}, \boldsymbol{J}_{T R}^{T}\right]$.

\section{Condition for motion planning}

\subsection{Kinematic aspects}

The kinematic problems to be solved for a motion planning are to determine a joint velocity for a given object velocity or vice versa[3],[9].

The manipulation system is said to be kinematically manipulable, for a given desired object velocity, $\dot{\boldsymbol{q}}$, if it is possible to find a joint velocity, $\dot{\boldsymbol{\theta}}$, to accommodate the object trajectory. This implies that $\boldsymbol{J}_{A} \in \Re^{\left(2 n_{R}+n_{S}\right) \times n_{\theta}}$ is full rank and $2 n_{R}+n_{S} \leq n_{\theta}$.

On the other hand, the manipulation system is said to be kinematically determinate, if for a feasible joint velocity, $\dot{\boldsymbol{\theta}}$, the object velocity, $\dot{\boldsymbol{q}}$, is uniquely determined. This implies that $\boldsymbol{G}_{A} \in \Re^{3 \times 2 n_{R}+n_{S}}$ is full column rank and $2 n_{R}+n_{S} \geq 3$.

\subsection{Dynamic aspects}

The dynamic problems to be solved for a motion planning are to determine a joint torque for a given object acceleration or vice versa.

The manipulation system is said to be dynamically manipulable, for a given object acceleration, $\ddot{q}$, if it is possible to find a joint torque, $\tau$, to accommodate the object trajectory. This implies that (i) $\boldsymbol{G}_{A \mu} \in \Re^{3 \times 2 n_{R}+n_{S}}$ is full rank and $2 n_{R}+n_{S} \geq 3$ such that there exists $\boldsymbol{f}_{A}$ for any inertial and external force, (ii) $\boldsymbol{J}_{A \mu}^{T} \in \Re^{n_{\theta} \times\left(2 n_{R}+n_{S}\right)}$ is full rank such that there exists $\tau$ which can control each component of $\boldsymbol{f}_{A}$, and (iii) $\boldsymbol{J}_{A}$ is full rank such that $\ddot{\boldsymbol{\theta}}$ can be determined for a given object acceleration, $\ddot{\boldsymbol{q}}$, from the acceleration kinematics,

$$
\boldsymbol{G}_{A}^{T} \ddot{\boldsymbol{q}}-\boldsymbol{J}_{A} \ddot{\boldsymbol{\theta}}+\dot{\boldsymbol{G}}_{A}^{T} \dot{\boldsymbol{q}}-\dot{\boldsymbol{J}}_{A} \dot{\boldsymbol{\theta}}=\mathbf{0}
$$

which can be given by differentiating Equation(4).

On the other hand, the manipulation system is said to be dynamically determinate if a dynamics model uniquely predict an object acceleration and a contact force for a given joint torque. Therefore, the problem can be reduced to a linear complementarity problem (LCP) that can be formulated as[8],[14]

$$
\boldsymbol{x} \geq \mathbf{0}, \boldsymbol{y}=\boldsymbol{A x}+\boldsymbol{b}, \boldsymbol{x}^{T} \boldsymbol{y}=\mathbf{0}
$$

where

$$
\boldsymbol{A}=\left[\boldsymbol{G}_{A}^{T}-\boldsymbol{J}_{A}\right]\left[\begin{array}{cc}
\boldsymbol{M}_{o} & \mathbf{0} \\
\mathbf{0} & \boldsymbol{M}_{a}
\end{array}\right]\left[\begin{array}{c}
\boldsymbol{G}_{A \mu} \\
-\boldsymbol{J}_{A \mu}^{T}
\end{array}\right]
$$

The LCP has an unique solution for any joint torque if and only if the matrix $\boldsymbol{A}$ is $\boldsymbol{P}$-matrix[2]. Therefore dynamically determinate requires that the matrix $\boldsymbol{A}$ in Equation(7) belongs to the class of $\boldsymbol{P}$-matrix.

From the result mentioned above, we can get the following kinematical and dynamical conditions for the motion planning of whole arm manipulation as:

$$
3 \leq 2 n_{R}+n_{S} \leq n_{\theta}
$$




$$
\begin{array}{r}
\operatorname{rank}\left(\boldsymbol{J}_{A}\right)=2 n_{R}+n_{S} \\
\operatorname{rank}\left(\boldsymbol{J}_{A \mu}\right)=2 n_{R}+n_{S} \\
\operatorname{rank}\left(\boldsymbol{G}_{A}\right)=3 \\
\operatorname{rank}\left(\boldsymbol{G}_{A \mu}\right)=3 \\
\boldsymbol{A} \in \boldsymbol{P} \text {-matrix class }
\end{array}
$$

Note that these conditions restrict the contact mode and the number of contact points.

\section{Instantaneous motion planning}

In this section we now develop the algorithms for determining joint torques that will realize a specified object trajectory at every instant, satisfying the conditions Equations $(8) \sim(13)$. In the next section, we will relax the conditions on the contact mode and allow the modes to change.

We have the general problem that joint torques may not be unique for a specified object trajectory. When the system is dynamically manipulable with $2 n_{R}+n_{S}<n_{\theta}$ for a current contact mode, there are multiple solutions to $\ddot{\theta}$ for $\ddot{\boldsymbol{q}}$. Using the generalized inverse of $\boldsymbol{J}_{A}$, the minimum norm of $\ddot{\theta}$ can be uniquely determined for $\ddot{\boldsymbol{q}}$ from Equation(5) as;

$$
\ddot{\boldsymbol{\theta}}=\boldsymbol{J}_{A}^{+}\left(\boldsymbol{G}_{A}^{T} \ddot{\boldsymbol{q}}+\dot{\boldsymbol{G}}_{A}^{T} \dot{\boldsymbol{q}}-\dot{\boldsymbol{J}}_{A} \dot{\boldsymbol{\theta}}\right)
$$

When the wrench matrix, $\boldsymbol{G}_{A \mu}$, is over-constrained, there are multiple solutions to a contact force for a given object acceleration. We can determine the minimum norm of the contact force, $f_{A}$, which satisfies Coulomb's friction law by solving the following quadratic programming problem,

$$
\begin{aligned}
\text { minimize } & 1 / 2 \boldsymbol{f}_{A}^{T} \boldsymbol{f}_{A} \\
\text { subject to } & \text { Eqs.(1)and(3) }
\end{aligned}
$$

The algorithm for an instantaneous motion planning is shown below. For a given contact mode, $\mathcal{M}$, and object acceleration, $\ddot{\boldsymbol{q}}(t)$, the joint acceleration, $\ddot{\boldsymbol{\theta}}(t)$, and contact force, $f_{A}(t)$, are determined using Equations(14) and (15), respectively. The joint torque, $\boldsymbol{\tau}(t)$ can be determined by substituting $\ddot{\boldsymbol{\theta}}$ and $\boldsymbol{f}_{A}$ into the arm dynamic equation (2). The states, $\boldsymbol{q}$ and $\dot{\boldsymbol{q}}$, and $\boldsymbol{\theta}$ and $\dot{\boldsymbol{\theta}}$ at next time step are obtained by an integration of $\ddot{\boldsymbol{q}}(t)$ and $\ddot{\boldsymbol{\theta}}(t)$.

\section{Global motion planning}

\subsection{Hybrid system}

The continuous state space, $\mathcal{X}$, of manipulation systems consists of multiple partitions, $\mathcal{X}_{k}\left(\mathcal{M}_{k}\right)$, corresponding to a contact mode, $\mathcal{M}_{k} \in \mathcal{M} \subseteq[R R, R S, 2 S R, \cdots]$,

$$
\mathcal{X}=\bigcup_{k}^{m} \mathcal{X}_{k}\left(\mathcal{M}_{k}\right)
$$

The continuous states, $\boldsymbol{q}, \dot{\boldsymbol{q}}, \boldsymbol{\theta}$ and $\dot{\boldsymbol{\theta}}$, of each $\mathcal{X}_{k}$ is governed by different dynamic and kinematic equations. These states change continuously between partitions. Since the whole arm manipulation consists of a sequence of partitions of state space as well as a continuous trajectory of the system, we call such a system hybrid system. Figure 2 illustrates the hybrid system. $\boldsymbol{x}(0)$ and $\boldsymbol{x}\left(t_{f}\right)$ denote initial and goal states, respectively. The trajectory, $\alpha$, passes through from $\mathcal{X}_{1}, \mathcal{X}_{2}$, to $\mathcal{X}_{5}$ while the trajectory, $\beta$, passes on another partitions. The black circles indicate the switching between the partitions.

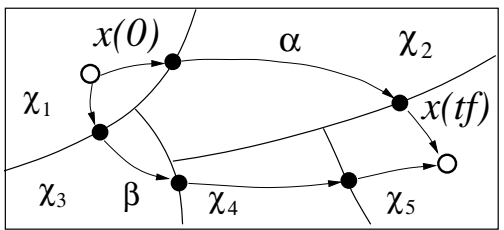

Figure 2: Manipulation system with switching contact mode.

We now define the motion planning problem for the whole arm manipulation as, "given an initial object state, $\boldsymbol{q}(0), \dot{\boldsymbol{q}}(0)$ and a goal object state, $\boldsymbol{q}\left(t_{f}\right), \dot{\boldsymbol{q}}\left(t_{f}\right)$, it is to find a trajectory which connects initial state with final state, switching rolling and sliding contacts repeatedly." Each trajectory on the interval $t \in\left[t_{j}, t_{j+1}\right], 0<t_{1}<\cdots<$ $t_{N+1}=t_{f}$ corresponds to each $\mathcal{X}_{j}\left(\mathcal{M}_{j}\right)$. The trajectory switches from $\mathcal{X}_{j}$ to $\mathcal{X}_{j+1}$ at the switching time, $t_{j+1}$. Specified an object trajectory, the motion planning problem consists of four components: (i) the number of switches, $N$, (ii) the switching time, $\left\{t_{j}\right\}_{j=1}^{N}$, (iii) the sequence of contact mode, $\left\{\mathcal{M}_{j}\right\}_{j=0}^{N}$, (iv) the state trajectory and joint torque trajectory on each interval $\left[t_{j}, t_{j+1}\right]$. Note that the object trajectory is assumed to be specified. But one can imagine a motion editing system where this trajectory is modified interactively, or an outer loop that modifies the object trajectory according to a cost function. The algorithm for the four components are organized in the flow chart in Figure 3. We will describe each of these components and the organization next.

\subsection{Algorithm}

It is very difficult to find all components of motion planning problem for the hybrid system because the planning requires exploring the high dimensional search space. We can find an appropriate solution by assigning an object trajectory and a sequence of switching time. The planning problem can be reduced to the problem which finds the sequence of contact modes and joint torque trajectory.

The proposed manipulation planning, as shown in Figure 3, consists of (i) generation of an object sub-trajectory on $\left[t_{j}, t_{j+1}\right]$, (OBJ_TRAJ_PLANNER), (ii) search for feasible contact modes, calculation of joint torque which is feasible to manipulate an object along the given object sub-trajectory on $\left[t_{j}, t_{j+1}\right]$, generation of subgoals, and addition of feasible solutions into a list, $\mathcal{L T}_{j}$, (MOTION_SUBINTERVAL), (iii) selection of desirable trajec- 


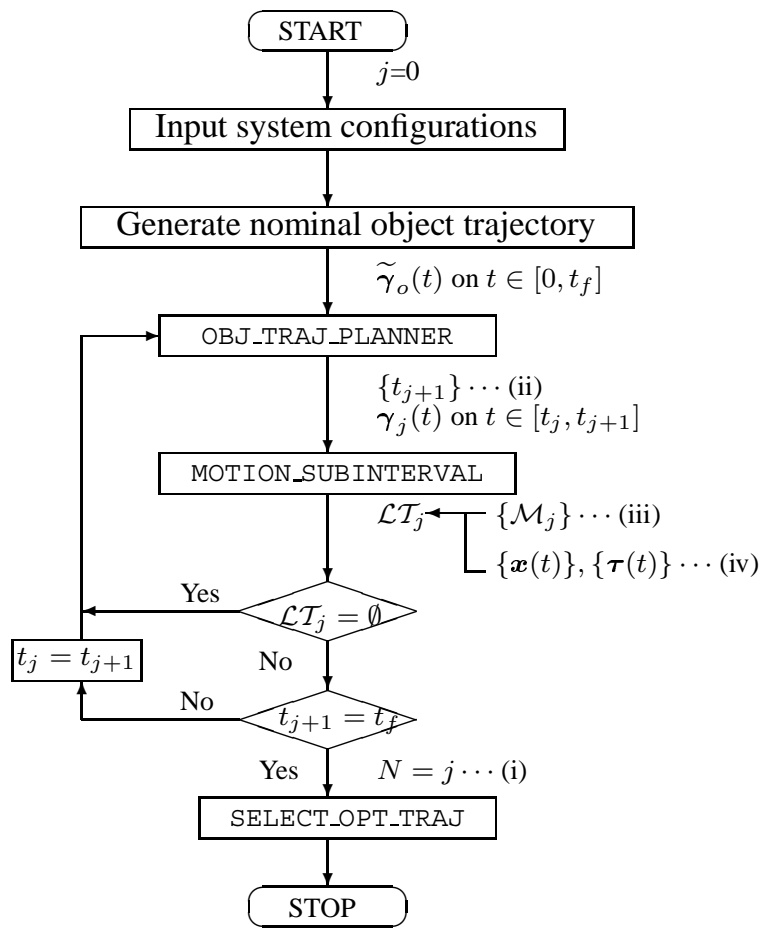

Figure 3: An algorithm of motion planning.

tory, (SELECT_OPT_TRAJ). (i) and (ii) are iterated whenever the switching time is updated until a final time, $t_{f}$, and feasible subgoals are generated. Finally, the function SELECT_OPT_TRAJ gives the desirable sequence of contact mode and joint torque trajectory based on the cost function,

$$
f_{\text {cost }}=\sum_{i=0}^{N} \int_{t_{i}}^{t_{i+1}} P(\boldsymbol{x}, \boldsymbol{\tau}) d t
$$

Figure 4 shows the iterative process of system state exploration using this algorithm. At each switching time, the feasible contact modes are determined and the subgoals are generated. Note that the states in each subgoal are different because the joint and contact states are different though same object trajectory is given.

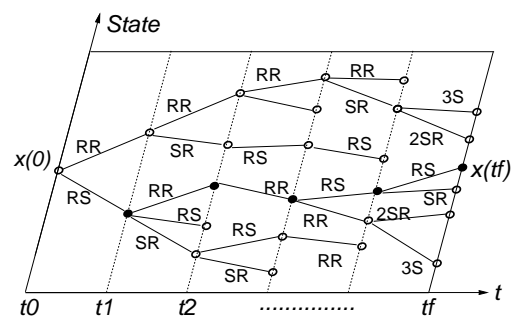

Figure 4: Iterative process of state expansion with changing contact modes.

The function OBJ_TRAJ_PLANNER provides the next switching time, $t_{j+1}$, and the object sub-trajectory, $\gamma_{j}(t), t \in\left[t_{j}, t_{j+1}\right]$. As shown in Figure 5, the next switching time, $t_{j+1}$, is assigned randomly for a current switching time, $t_{j}$. Then the object sub-trajectory, $\gamma_{j}(t)$, which satisfies the following boundary conditions,

$$
\gamma_{j}\left(t_{j}\right)=\widetilde{\gamma}_{o}\left(t_{j}\right)
$$

$$
\begin{array}{r}
\gamma_{j}\left(t_{j+1}\right)=\widetilde{\gamma}_{o}\left(t_{j+1}\right) \\
\dot{\gamma}_{j}\left(t_{j}\right)=\dot{\gamma}_{j}\left(t_{j+1}\right)=0
\end{array}
$$

is generated using a nominal object trajectory, $\widetilde{\gamma}_{o}(t), t \in$ $\left[0, t_{f}\right]$ which is usually known a priori as a desirable rough trajectory. The first and second equations in Equation(18) show that the object sub-trajectory, $\gamma_{j}$, goes from object states, $\boldsymbol{q}\left(t_{j}\right)$ and $\dot{\boldsymbol{q}}\left(t_{j}\right)$ to $\boldsymbol{q}\left(t_{j+1}\right)$ and $\dot{\boldsymbol{q}}\left(t_{j+1}\right)$ on the nominal trajectory. The third equation implies that a relative tangential velocity at each contact point makes zero at each switching time so that we can assign any contact mode at switching time. The idea of generating an object trajectory by giving a switching time randomly, rather that directly sampling the state space of the object, make the exploration quickly over the state space and the planner find feasible solutions easily[5].

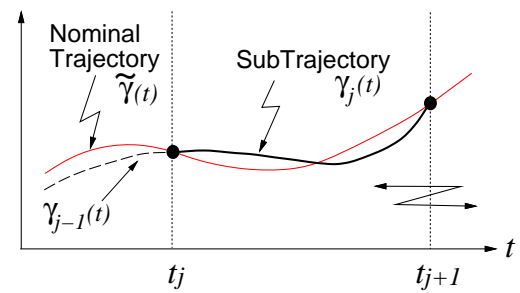

Figure 5: Planning for object trajectory based on randomized approach.

The algorithm of MOTION_SUBINTERVAL is shown below. Whenever the switching time is updated, a contact mode is assigned among $2^{n_{c}}$ combinations of contact modes, and the instantaneous manipulation planning shown in the previous section is iterated at every instant on $\left[t_{j}, t_{j+1}\right]$ for the assigned $\gamma_{j}$. The feasible contact modes are searched which satisfy Equations $(8) \sim(13)$. If we can find feasible solutions on $\left[t_{j}, t_{j+1}\right]$, then the subgoals are generated, and $t_{j+1}, \boldsymbol{q}\left(t_{j+1}\right), \theta\left(t_{j+1}\right), \mathcal{M}_{j}$, and cost, $f_{\text {cost }_{j}}=\int_{t_{j}}^{t_{j+1}} P(x, \tau) d t$, are stored into the list $\mathcal{L}_{j}$. If there is no feasible solution, another object sub-trajectory is assigned again.

\section{Simulation results}

Consider to plan a whole arm manipulation in order to lift up an object toward a palm. As shown in Figure 6, the fingers of a two 2-DOF fingered hand with a fixed palm are used to manipulate an elliptical object in the vertical plane with rolling and/or sliding contacts. Each 2nd link of the both arms has the contact with the object at the initial time. We assume that the breaking contacts are not occurred once the links contact the object. $\Sigma_{B}$ is the base frame placed in the midpoint between the two joints in the palm and $\Sigma_{i}$ is the coordinate frame attached to the $i$ th contact point.

In this example, the following constraints about the number of rolling and sliding contacts are imposed:

$$
\begin{aligned}
& n_{R}+n_{S} \leq 4 \\
& 3 \leq 2 n_{R}+n_{S} \leq 4
\end{aligned}
$$




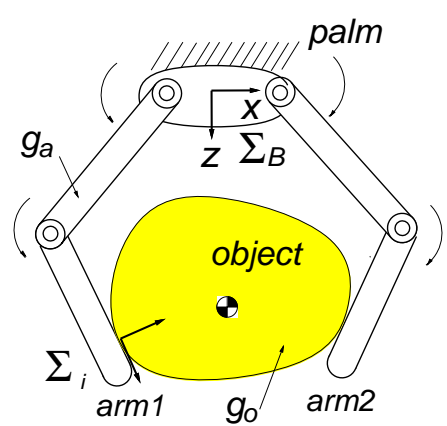

Figure 6: A planner whole arm grasp.

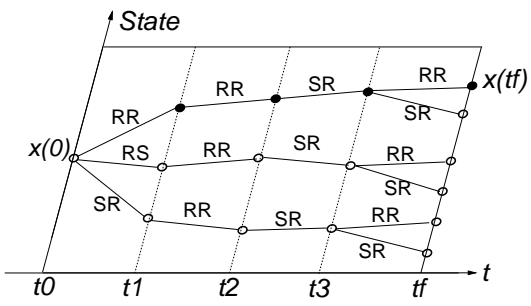

Figure 7: Simulation results of state exploration.

The first constraint says that the total number of contacts between the object and the arms is less than four because of the assumption that the each link of each arm has one contact point. The second constraint is a condition for the motion planning which is obtained by substituting $n_{\theta}=4$ into Equation(8). The feasible contact mode set, $\mathcal{M}$, which satisfies these conditions is $\mathcal{M} \subseteq\{R R, R S, S R, 2 S R, R 2 S, 2 S S, S 2 S, 2 S 2 S\}$, where the first and second letter denotes the contact modes for the arm 1 and arm2, respectively, and the figure denotes the number of the contact mode. $R S$ indicates the combination of a rolling contact at the 2 nd link of arm 1 and a sliding contact at the 2 nd link of arm 2 .

The object has a major axis of $0.37 \mathrm{~m}$ and its minor axis is $0.30 \mathrm{~m}$. The mass of the object is $3.00 \mathrm{~kg}$, and the moment of inertia about the center of mass is $4.25 \times 10^{-2} \mathrm{~kg} \cdot \mathrm{m}^{2}$. The fixed palm of the hand is $0.20 \mathrm{~m}$ long. The length of each finger link is $0.30 \mathrm{~m}$. The mass of the finger link is $0.5 \mathrm{~kg}$ with a moment of inertia of $1.67 \times 10^{-3} \mathrm{~kg} \cdot \mathrm{m}^{2}$. The kinetic and static coefficient of friction are both $\mu_{i S}=\mu_{i R}=0.2$.

The manipulation task is to rotate the object from 20 degree to 0 degree, and to translate it from $0.30 \mathrm{~m}$ to $0.15 \mathrm{~m}$ in the $z$ direction in two seconds. We can get six feasible solutions with three switchings, as shown in Figure 7. The feasible solution, whose transition of contact modes is from $R R, R R, S R$, to $R R$, is selected because of the minimum consumption of the joint driven torque.

The snap shots of the selected solution are shown in Figures 8 . The contact modes are denoted on the top of each snap shot when the contact mode is switched. The 2nd links with rolling and sliding contacts are drawn by the thick and thin line, respectively. The lines with arrow denote the contact force, the object linear and angular acceleration.

Figure 9 shows the nominal object trajectory and the
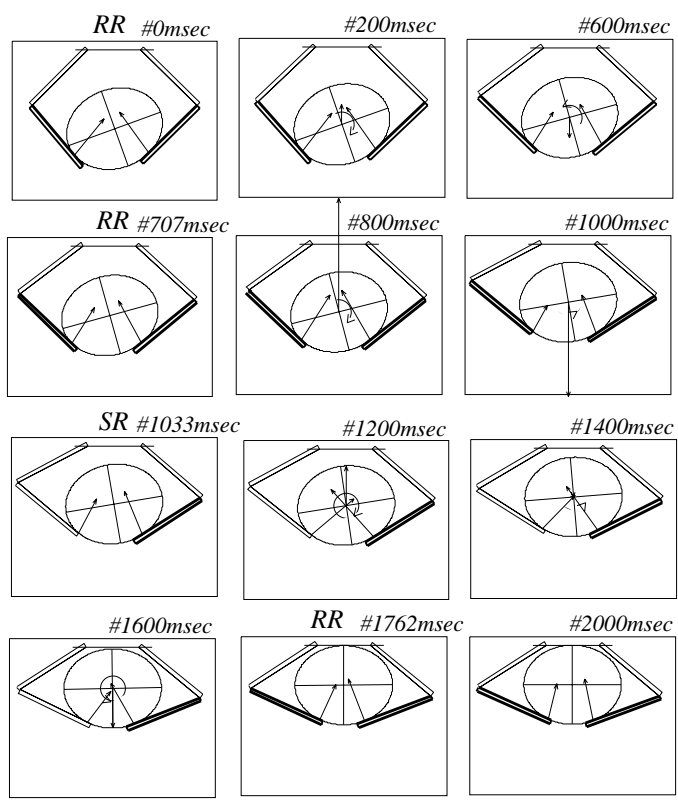

Figure 8: Snap shots of whole arm manipulation which is lifting an object.
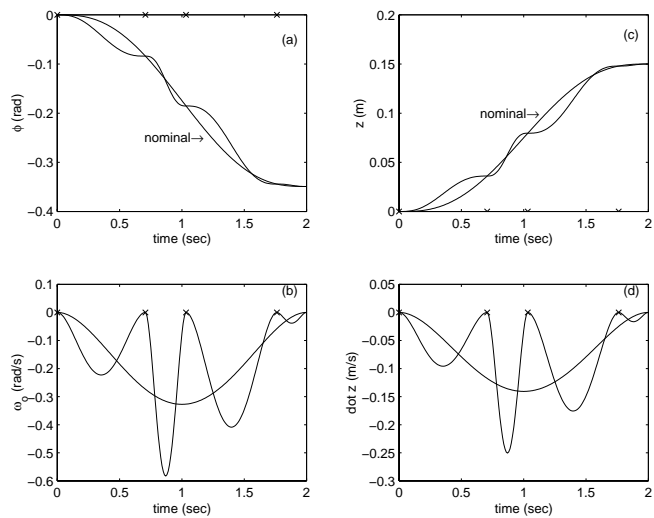

Figure 9: Nominal object trajectory and generated subtrajectory using randomized technique. (a) and (b) show the trajectory of the orientation and angular velocity, and (c) and (d) show trajectory of position and velocity in the $z$ direction. $\times$ indicates the switching. 
generated sub-trajectory using randomized technique. A fifth polynomial is used to interpolate the object subtrajectory on each interval. The velocities at each switching time become zero due to the boundary conditions Equation(18).

\section{Concluding remarks}

This paper presents the mathematical conditions and algorithm for the whole arm manipulation planning with switching contact modes. Changing the contact mode allows to extend the feasibility of manipulation. Motion planning for such a system should consider changing the dynamics and kinematics according to the contact modes. We showed the conditions about kinematic and dynamics aspects to be satisfied for the motion planning. The conditions give the restriction for feasible contact modes and the number of contact points.

The planning problem is simplified by giving the object trajectory using a randomized technique. It is not always guaranteed to find a trajectory whenever one exists. Though the algorithm is not complete, we could find the solution trajectory efficiently compared to the direct sampling approach. The main limitation of the proposed approach is that we must specify an appropriate nominal object trajectory a priori such that feasible solutions could be found by the algorithm.

\section{Acknowledge}

It is a pleasure to thank Dr. Vijay Kumar, University of Pennsylvania, for helpful comments.

\section{References}

[1] M.J.Cherif, K.K.Gupta. Planning quasi-static fingertip manipulations for reconfiguring objects. IEEE Transaction on Robotics and Automation, 15(5):837-848, 1999.

[2] R.W.Cottle, J.Pang, R.E.Stone. The Linear Complementarity Problem. Academic Press, 1992.

[3] L.Han, J.C.Trinkle. The instantaneous kinematics of manipulation. In Proc. of IEEE Int. Conf. on Robotics and Automation, 1998.

[4] M.Kaneko, M.Higashimori, T.Tsuji Transition stability of enveloped objects. In Proc. of IEEE Int. Conf. on Robotics and Automation, 1998.

[5] S.M.LaValle, J.J.Kuffner,Jr. Randomized kinodynamic planning. In Proc. of IEEE Int. Conf. on Robotics and Automation, 1999.

[6] M.D.Lemmon, K.X.He, I.Markovsky. Supervisory hybrid systems. IEEE Control Systems, August:42-55, 1999.

[7] Z.Li, J.F.Canny, S.Sastry. On motion planning for dextrous manipulation, part I: the problem formulation. In Proc. of IEEE Int. Conf. on Robotics and Automation, pages 775780, 1989.
[8] P.Lötstedt. Coulomb friction in two-dimensional rigid body systems, Zeitschrift Für Angewandte Mathematik und Mechanik, 61:605-615, 1981.

[9] D.J.Montana. The kinematics of multi-fingered manipulation. IEEE Transaction on Robotics and Automation, 11(4):491-503, 1995.

[10] T.Omata Rigid body analysis of the indeterminate grasp force in power grasps. In Proc. of IEEE Int. Conf. on Robotics and Automation, 1996.

[11] P.Song, M.Yashima, V.Kumar. Dynamic simulation for grasping and whole arm manipulation. In Proc. of IEEE Int. Conf. on Robotics and Automation, 2000.

[12] J.C.Trinkle, J.Abel, R.Paul. An investigation of frictionless enveloping grasping in the plane. Int. Journal of Robotics Research, 7(3):33-51, 1988.

[13] J.C.Trinkle, J.J.Hunter. A framework for planning dextrous manipulation. In Proc. of IEEE Int. Conf. on Robotics and Automation, pages 1245-1251, 1991.

[14] J.C.Trinkle, J.S.Pang, S.Sudarsky, G.Lox. On dynamics multi-rigid-bidy contact problems with Coulomb friction. Zeitschrift Für Angewandte Mathematik und Mechanik, 77(4):267-279, 1997.

[15] M.Yashima, H.Yamaguchi. Control of whole finger manipulation utilizing frictionless sliding contact -theory and experiment-. Mechanism and Machine Theory, 34:12551269, 1999.

[16] M.Zefran, J.P.Desai, V.Kumar. Continuous motion plans for robotics systems with changing dynamic behavior. In Algorithmic Foundations of Robotics, 1996.

[17] X.Zhang,Y.Nakamura,K.Gota, K.Yoshimoto. Robustness of power grasp. In Proc. of IEEE Int. Conf. on Robotics and Automation, pages 2828-2835, 1994. 